\title{
Management of rhinosinusitis during pregnancy: systematic review and expert panel recommendations*
}

\author{
Devyani Lal', Ameya A. Jategaonkar², Larry Borish³, Linda R. Chambliss4, \\ Sharon H. Gnagi', Peter H. Hwang', Matthew A. Rank', James A. Stankiewicz', \\ Valerie J. Lund ${ }^{8}$ \\ ' Department of Otolaryngology - Head \& Neck Surgery, Mayo Clinic in Arizona, Phoenix, AZ, USA \\ ${ }^{2}$ University of Arizona College of Medicine - Phoenix, Phoenix, AZ, USA \\ ${ }^{3}$ Departments of Medicine and Microbiology, University of Virginia, Charlottesville, VA, USA \\ ${ }^{4}$ Division of Maternal Fetal Medicine, St. Joseph's Hospital and Medical Center, Phoenix, AZ, USA \\ ${ }^{5}$ Department of Otolaryngology - Head \& Neck Surgery, Stanford University Medical Center, Stanford, CA, USA \\ ${ }^{6}$ Division of Allergy, Asthma, and Clinical Immunology, Mayo Clinic in Arizona, Phoenix, AZ, USA \\ ${ }^{7}$ Department of Otolaryngology - Head \& Neck Surgery, Loyola University Medical Center, Maywood, IL, USA \\ ${ }^{8}$ Royal National Throat, Nose and Ear Hospital, University College London Hospitals, London, United Kingdom
} Rhinology 54: 99-104, 2016

August 19, 2015

Accepted: November 12, 2015

\begin{abstract}
Background: Management of rhinosinusitis during pregnancy requires special considerations.
\end{abstract}

Objectives: 1. Conduct a systematic literature review for acute and chronic rhinosinusitis (CRS) management during pregnancy. 2. Make evidence-based recommendations.

Methods: The systematic review was conducted using MEDLINE and EMBASE databases and relevant search terms. Title, abstract and full manuscript review were conducted by two authors independently. A multispecialty panel with expertise in management of Rhinological disorders, Allergy-Immunology, and Obstetrics-Gynecology was invited to review the systematic review. Recommendations were sought on use of following for CRS management during pregnancy: oral corticosteroids; antibiotics; leukotrienes; topical corticosteroid spray/irrigations/drops; aspirin desensitization; elective surgery for CRS with polyps prior to planned pregnancy; vaginal birth versus planned Caesarian for skull base erosions/ prior CSF rhinorrhea.

Results: Eighty-eight manuscripts underwent full review after screening 3052 abstracts. No relevant level 1, 2, or 3 studies were found. Expert panel recommendations for rhinosinusitis management during pregnancy included continuing nasal corticosteroid sprays for CRS maintenance, using pregnancy-safe antibiotics for acute rhinosinusitis and CRS exacerbations, and discontinuing aspirin desensitization for aspirin exacerbated respiratory disease. The manuscript presents detailed recommendations.

Conclusions: The lack of evidence pertinent to managing rhinosinusitis during pregnancy warrants future trials. Expert recommendations constitute the current best available evidence.

Key words: pregnancy, sinusitis, paranasal sinus diseases, drug therapy, guideline

\section{Introduction}

Approximately 29.6 million adults in the United States suffer from sinusitis, with 11 million suffering from chronic rhinosinusitis $(C R S)^{(1)}$. The multicenter GA2LEN study showed that amongst lifetime nonsmokers, women were at a greater risk of being affected by chronic rhinosinusitis than men ${ }^{(2)}$. Rhinologic mani- festations of pregnancy have been previously described, and the commonly discussed conditions include rhinitis of pregnancy, epistaxis and certain tumors such as pyogenic granuloma ${ }^{(3-7)}$. Sporadic case reports describe complications that may arise from rhinosinusitis during pregnancy ${ }^{(8,9)}$. However, management of rhinosinusitis during pregnancy is poorly described 
in the literature ${ }^{(3)}$. Furthermore, clinical practice guidelines on chronic rhinosinusitis from the American Academy of Otolaryngology-Head \& Neck Surgery and the European Rhinologic Society also do not offer structured guidelines to Otolaryngologists on managing rhinosinusitis in the pregnant patient ${ }^{(10,11)}$. Therefore, there is a need for reviewing the literature to examine the evidence for managing rhinosinusitis and its associated rhinologic comorbidities during pregnancy.

Here we study management of rhinosinusitis (RS) during pregnancy so as to provide recommendations for evidence-based management and best practices.

\section{Methods}

Data sources and literature search

A systematic review of the English language literature was conducted using the MEDLINE (1966-2012) and EMBASE (19802012) databases. The search titles, abstract screening, and full manuscript review was performed independently by two authors (DL and AAJ). The level of evidence was graded according to the Oxford Centre of Evidence Based Medicine 2009 criteria (12).

A comprehensive search strategy using PRISMA (Preferred Reporting Items for Systematic Reviews and Meta-Analyses) guidelines was developed (Figure 1). Randomized controlled trials (RCTs), comparative studies, observational studies, case series, and case reports were evaluated for eligibility.

Studies related to rhinitis and rhinosinusitis were both evaluated, as these conditions may be difficult to distinguish in the absence of radiologic testing (computerized tomography) or rhinoscopy. Studies that comprised primary research on pregnant human females were included. Studies were excluded if they consisted of animal or in vitro research, or if they were review articles with no primary research data.

\section{Search strategy}

The search strategy was chosen in consultation with a senior librarian at the Mayo Clinic (LM) as follows: "pregnancy" [MeSH] AND "female" [MeSH] OR "gender" [MeSH] OR "sinusitis" [MeSH] OR sinusitis OR "rhinitis" [MeSH] OR "rhinosinusitis" [MeSH] OR "corticosteroid" [MeSH] OR "corticosteroids" [MeSH] OR "cerebrospinal fluid" OR "CSF" [MeSH] OR "skull Base" [MeSH] OR "aspirin" [MeSH]. English language publications from January 1966 to December 2014 (MEDLINE) or January 1988 to December 2014 (EMBASE) were scrutinized. The search titles were initially independently screened by two authors (DL and AAJ) to select abstracts that broadly met inclusion criteria. Next, full manuscripts were retrieved based on this independent review and again reviewed independently by the two authors. Additional studies were included for review based on the references and citations of the full manuscripts. Duplicated articles and those not meeting study criteria after full review were excluded.

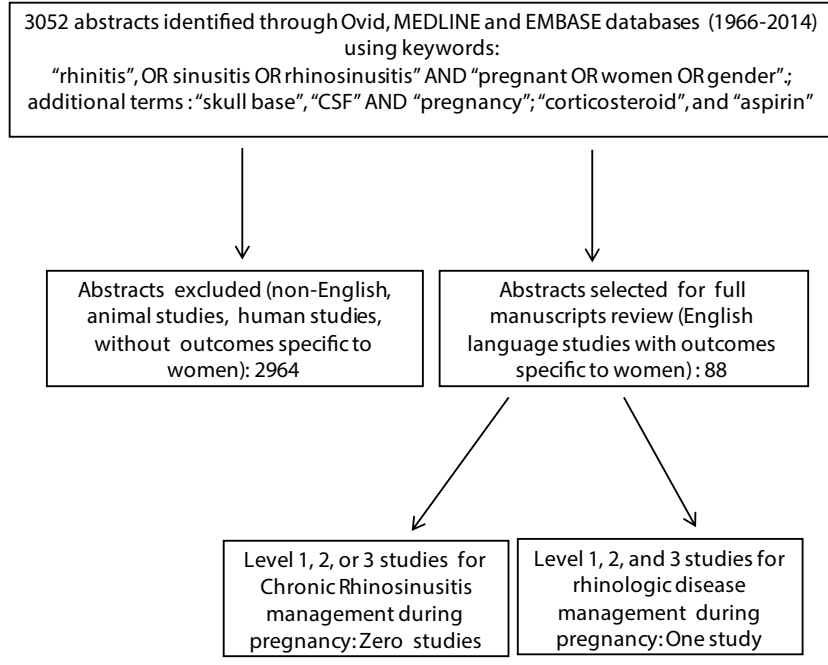

Figure 1. Search methodology and results of the systematic review.

\section{Expert panel recommendations}

An international, multidisciplinary expert panel was then created. Experts from the fields of Rhinology, Allergy-Immunology, and Obstetrics-Gynecology were invited. They were selected based on their expertise in a) systematic reviews and evidencebased management of rhinosinusitis ( $\mathrm{DL}, \mathrm{PHH}, \mathrm{VJL})$, its complications and associated medico-legal aspects (DL, JAS, VJL), b) expertise in medication/therapeutics of CRS, allergy \& asthma (DL, PHH, LB, MAR, JAS, VJL), as well as in c) obstetrics \& perinatal issues (LRC). Each expert was provided with the full results of the systematic review, and asked to provide their input and expertise on specific questions (Table 1). These expert opinions were then reviewed and synthesized into an expert panel recommendation by the first and senior authors. Next, these recommendations were shared with all experts, collectively and iteratively reviewed, and a final expert panel opinion was formalized. The final authorship thus included four Rhinologists (DL, PHH, JAS and VJL), two experts in Allergy, Immunology and Asthma (LB and MAR) and an expert in Maternal and Fetal Medicine (LRC).

\section{Results}

\section{Results Summary}

The literature search yielded 3052 abstracts. After initial screening, 88 full manuscripts were reviewed. No studies related to CRS management during pregnancy that were levels 1 , 2, or 3 were found. A two-author, single expert opinion on management of rhinologic disease during pregnancy was found ${ }^{(5)}$. Given the lack of any studies on CRS management during pregnancy, expert panel recommendations were synthesized and are presented below.

Table 2 presents the pregnancy risk categories for therapeutic agents. 
Table 1. Queries presented to experts with regard to chronic rhinosinusitis (crs) management during pregnancy.

\begin{tabular}{|c|c|}
\hline $\begin{array}{l}\text { Question } \\
\text { Number }\end{array}$ & Clinical Question \\
\hline 1 & $\begin{array}{l}\text { Use of oral corticosteroids for CRS exacerbations during } \\
\text { pregnancy? }\end{array}$ \\
\hline 2 & $\begin{array}{l}\text { Use of topical corticosteroids for CRS maintenance the- } \\
\text { rapy during pregnancy? }\end{array}$ \\
\hline 3 & $\begin{array}{l}\text { Use of oral antibiotics for acute rhinosinusitis, acute } \\
\text { exacerbations of CRS, and CRS maintenance during } \\
\text { pregnancy? }\end{array}$ \\
\hline 4 & $\begin{array}{l}\text { Use of anti-leukotrienes for CRS maintenance therapy in } \\
\text { the pregnant patient? }\end{array}$ \\
\hline 5 & $\begin{array}{l}\text { Use of systemic and topical decongestants, antihistamines } \\
\text { and immunotherapy for CRS management in pregnancy? }\end{array}$ \\
\hline 6 & $\begin{array}{l}\text { What "Routine" maintenance therapy do you recommend } \\
\text { for CRS during pregnancy? }\end{array}$ \\
\hline 7 & $\begin{array}{l}\text { Aspirin desensitization therapy during pregnancy for } \\
\text { aspirin exacerbated respiratory disease (AERD)? }\end{array}$ \\
\hline 8 & $\begin{array}{l}\text { Vaginal Births versus planned Caesarian section for } \\
\text { those with large skull base erosions secondary to CRS, } \\
\text { secondary to skull base resection, or patients with benign } \\
\text { intracranial HTN with history of CSF rhinorrhea? }\end{array}$ \\
\hline 9 & $\begin{array}{l}\text { Performance of sinus surgery in patients with recurrent, } \\
\text { recalcitrant CRS with polyps prior to conception of a plan- } \\
\text { ned pregnancy? }\end{array}$ \\
\hline
\end{tabular}

1. Use of oral corticosteroids for CRS exacerbations during pregnancy?

Evidence Level: Zero; no studies found; one review article outlined the pregnancy risks associated with antibiotics, antihistamines, decongestants, and intranasal steroids.

Expert Panel Recommendations: Corticosteroids (CCS) in short bursts may be safe after the first trimester. Use is better justified in severe CRS, especially if causing exacerbation of asthma. Consultation with patient's obstetrician is recommended.

Underlying Values \& Preferences: Places high value on maternal and fetal safety over sinonasal symptom control.

Remarks: Based upon the asthma literature, oral CCS use would be expected to be associated with slightly increased risk for cleft lip with or without cleft palate ${ }^{(13)}$, increased incidence of preeclampsia, and the delivery of both preterm and low birth weight infants ${ }^{(14,15)}$. The risks of CCS use are outweighed by risks of undertreated asthma, but this justification is less so in CRS and clinical judgment must be exercised. First trimester use has the greatest risk of potential teratogenicity. CCS cause hyperglycemia and can lead to/worsen diabetes, causing additional maternal-fetal risks. Patients should undergo diabetes testing prior to use, especially if a longer course of corticosteroids is being considered. The American Academy of Pediatrics considers oral steroids to be compatible with breastfeeding.

2. Use of topical corticosteroids for CRS maintenance the-
Table 2. Categories of pharmaceutical agents assessing risk for use in pregnancy ${ }^{(30)}$

\section{Category}

\section{Description}

A Adequate, well-controlled studies in pregnant women have not shown an increased risk of fetal abnormalities.

B Animal studies have revealed no evidence of harm to the fetus; however, there are no adequate and well-controlled studies in pregnant women.

Or,

Animal studies have shown an adverse effect, but adequate and well-controlled studies in pregnant women have failed to demonstrate a risk to the fetus.

C Animal studies have shown an adverse effect, or no animal studies have been conducted, and there are no adequate and well-controlled studies in pregnant women.

D Adequate, well-controlled, or observational studies in pregnant women have demonstrated a risk to the fetus. However, the benefits of therapy may outweigh the potential risk.

X Adequate, well-controlled, or observational studies in animals or pregnant women have demonstrated positive evidence of fetal abnormalities. The use of the product is contraindicated in women who are or may become pregnant.

\section{rapy during pregnancy?}

Evidence Level: Zero; no studies on topical nasal steroids for CRS during pregnancy.

Expert Panel Recommendations: All modern nasal CCS should be safe to use for CRS maintenance during pregnancy at recommended doses including budesonide, fluticasone and mometasone. The off-label use of budesonide irrigations or CCS nasal drops is not recommended.

Underlying Values \& preferences: Places value on maternal sinonasal inflammation control and quality of life during pregnancy while prioritizing fetal safety.

Remarks: The only study of nasal corticosteroid spray use in pregnancy, conducted by Ellegard, et al., evaluated fluticasone propionate nasal sprays in 53 women with pregnancy rhinitis in a placebo-controlled randomized study ${ }^{(16)}$. Daily symptom scores and nasal peak expiratory flow, as well as acoustic rhinometry before and after treatment did not show any differences between the groups. No detectable influence on maternal cortisol as measured by morning S-cortisol and overnight 12-h$\mathrm{U}$-cortisol, or any difference in ultrasound measures of fetal growth or pregnancy outcome were reported. Another study was a single non-controlled, non-blinded case series of 21 patients who were given 27 intranasal injections of triamcinolone acetonide or prednisolone terburate for severe nasal obstruction during pregnancy ${ }^{(17)}$. Mabry reported symptom improvement, but there was no study of systemic CCS absorption or 
pregnancy outcomes. There is fairly good evidence for the safety of inhaled CCS in asthma during pregnancy ${ }^{(18-20)}$. Budesonide is category $B$ in pregnancy and remains the agent for which the preponderance of safety data exists ${ }^{(21)}$. Newer generation sprays have negligible systemic absorption and may be safe to use, but evidence that these medications during pregnancy are not associated with any untoward risks is lacking. This applies to usual pharmacologic doses. Budesonide is most widely studied; 50 micrograms in each nostril once or twice daily should be safe to use for CRS maintenance during pregnancy. Whether this extends to higher doses/potency steroids (e.g., $500 \mu \mathrm{g}$ budesonide or dexamethasone) applied as nasal drops (in Mygind's position) is unknown.

3. Use of oral antibiotics for acute rhinosinusitis, acute exacerbations of CRS, and CRS maintenance during pregnancy? Evidence Level: Zero; no studies on antibiotics for acute rhinosinusitis (ARS), acute exacerbations of CRS, and CRS maintenance. Expert Panel Recommendations: Oral antibiotics that do not harm the fetus may be used for ARS or acute exacerbations of CRS. Long-term macrolide or doxycycline use for CRS is not recommended during pregnancy. Penicillin and cephalosporin are the safest classes, and can be given when endoscopic evidence of purulence is present. Antibiotics that put the fetus at risk such as tetracyclines, aminoglycosides, trimethoprim-sulfamethaxazole and fluoroquinolones should not be used during pregnancy ${ }^{(22)}$. Underlying Values \& Preferences: Prioritizes fetal and maternal safety and places high value on appropriate treatment of bacterial rhinosinusitis.

\section{Anti-leukotriene medical therapy in CRS?}

Evidence Level: Zero; no studies on CRS.

Expert Panel Recommendations: Avoid anti-leukotrienes for CRS maintenance during pregnancy. However, montelukast can be continued/ initiated for recalcitrant asthma during pregnancy, especially in those with prior response.

Underlying Values \& preferences: Places high value on fetal and maternal safety.

Remarks: Given the lack of data regarding the efficacy of these drugs in CRS and lack of data regarding teratogenicity, the consensus of this panel was to avoid these drugs in treating CRS. Montelukast is not teratogenic in animals and the American Congress of Obstetricians and Gynecologists and the American College of Allergy recommend that it could be considered in recalcitrant asthma if the patient has shown a favorable response prior to pregnancy. Montelukast is a pregnancy category B drug and the manufacturer maintains a registry for patients who are exposed to montelukast in pregnancy. Montelukast is excreted in breast milk and there are no data on what this potential exposure could do to a nursing infant. However, the extensive metabolism and plasma protein binding are thought to limit exposure. About $1 \%$ of the drug passes into breast milk, and the benefits of breastfeeding overwhelm the risk of exposure. An additional consideration is to breastfeed prior to intake of medications to further limit exposure. 5-lipoxygenase inhibitors should be avoided in both pregnant and lactating mothers ${ }^{(23)}$.

\section{Use of systemic and topical decongestants, antihista-} mines and immunotherapy for CRS management in pregnancy?

Evidence Level: Zero: no studies on CRS.

Expert Panel Recommendations: Oral decongestants should not be used. First-generation antihistamines should be avoided given their sedative and anticholinergic properties. Allergen immunotherapy is likely safe to continue during pregnancy. However, initiation or buildup of immunotherapy should be not be conducted during pregnancy.

Underlying Values \& preferences: Prioritizes fetal and maternal safety over simple sinonasal symptom control.

Remarks: No studies assessed these drugs in CRS. Oral decongestants may increase the risk of fetal gastroschisis, and also contribute to hypertension ${ }^{(24)}$. Decongestants are also not effective in treating CRS. Toll et al. conducted an RCT of phenylpropanolamine for the treatment of pregnancy rhinitis ${ }^{(25)}$. They reported that it was more effective than placebo in treating the symptoms of patients. However, there was no difference in rhinometry. This study reported no adverse outcomes but did not report any fetal outcomes. It should be noted that phenylpropanolamine has been withdrawn from the US market. The greatest concern regarding allergen immunotherapy is the risk of anaphylaxis. Sublingual immunotherapy is also generally contraindicated during pregnancy ${ }^{(26)}$. Current recommendations for allergic rhinitis and asthma are that immunotherapy should be continued at the same dose but should not either be initiated nor should doses be increased during pregnancy.

6. What "Routine" maintenance therapy is recommended for CRS during pregnancy?

Evidence Level: Zero; no studies on CRS "maintenance" therapy in pregnancy.

Expert Panel Recommendations: Saline nasal rinses and a topical CCS nasal spray are likely suitable maintenance therapy for CRS during pregnancy.

Underlying Values \& preferences: Prioritizes fetal and maternal safety with value also placed to maternal quality of life and control of sinonasal inflammation.

Remarks: Studies in allergic rhinitis during pregnancy find saline nasal lavage and nasal CCS sprays safe and effective ${ }^{(11,27)}$.

\section{Aspirin therapy for aspirin exacerbated respiratory disease (AERD)?}

Evidence Level: Zero; no studies on AERD during pregnancy. 
Expert Panel Recommendations: Aspirin therapy for AERD should be discontinued during pregnancy.

Underlying Values \& preferences: Places high value on fetal and maternal safety.

Remarks: Aspirin is considered category $D$ in pregnancy and should be avoided. Aspirin and other NSAIDs pose unacceptable fetal risks, particularly premature closure of the ductus arteriosus. Aspirin itself has also been associated with intrauterine growth restriction and perinatal mortality. Patients who have previously been desensitized and are on maintenance aspirin therapy should discontinue aspirin prior to a planned pregnancy or as soon as possible after becoming pregnant.

8. Vaginal Births versus planned Caesarian section for those with large skull base erosions secondary to CRS, secondary to skull base resection, or patients with benign intracranial HTN with history of CSF rhinorrhea?

Evidence Level: Zero; no relevant studies.

Expert Panel Recommendations: Skull base erosions such as those discussed above are not contraindications for a normal delivery. Generally speaking, cesarean section should be reserved for the standard obstetric indications. However, a highly individualized approach must be undertaken, and ultimately the patient and the obstetrician should determine the route of delivery. The risks of intrapartum CSF leak may be much higher in large skull base resections for malignancies, rather than limited defects from CRS or standard transsphenoidal pituitary tumor resections. Underlying Values \& preferences: Places high value on fetal and maternal safety.

Remarks: One study from 1961 measured CSF pressures during labor and obstetric anesthesia and some case reports of intrapartum CSF leaks were also found ${ }^{(28,29)}$.

9. Performance of sinus surgery in patients with recurrent, recalcitrant CRS with polyps prior to planned pregnancy?

Evidence Level: Zero; no relevant studies.

Expert Panel Recommendations: Surgery may be considered prior to the pregnancy. In general, surgery that is not for a life threatening process should be avoided during pregnancy. Emergency surgery for complicated and acute/chronic sinusitis may be done with close anesthesia supervision. However, office procedures under local anesthesia may be some helpful alternatives in severely symptomatic CRS pregnant patients. These include polypectomy, indicated balloon sinuplasty, and turbinate surgery under local anesthesia.

Underlying Values \& preferences: Prioritizes fetal safety; value also placed to maternal quality of life.

\section{Discussion}

This comprehensive, systematic review did not find any level 1,2 , or 3 studies specific to the management of rhinosinusitis during pregnancy. While studies show an increase in sinonasal and allergic rhinitis symptoms during pregnancy, there are no studies on the evolution of CRS during pregnancy. This is a startling gap in our knowledge of the disease process during pregnancy, as well as in our ability to manage pregnant patients with CRS. Given that RS is believed to be more prevalent in females, and may affect them during the childbearing years, it is necessary to conduct relevant studies. Conducting interventional studies in pregnancy is fraught with medical and ethical dilemmas. Therefore, a start may be made by conducting large population-based studies such as those seen in the asthma literature.

Given the lack of interventional studies on management of RS during pregnancy, we found it necessary to generate expert panel recommendations (Level 5 evidence) to help guide management. Our multi-disciplinary panel of experts weighed the strength of evidence regarding specific interventions while balancing maternal health against potential fetal risks. These recommendations were given very careful consideration. Nevertheless, given the lack of high quality objective evidence, these are Grade D recommendations. These must be utilized and modified as determined after careful and thoughtful, individualized review of the pregnant patient, and in consultation with their Obstetrician.

\section{Author contributions}

DL conceived this study, independently performed the systematic review and significantly drafted the manuscript. AAJ independently performed the systematic review, significantly contributed to the manuscript draft and editing. LB is a member of the expert panel who helped construct the panel recommendations. He also performed a critical review of the manuscript. LRC is a member of the expert panel who helped construct the panel recommendations. She also performed a critical review of the manuscript. SHG contributed to the systematic review and performed a critical review of the manuscript. $\mathrm{PHH}$ is a member of the expert panel who helped construct the expert panel recommendations. He also performed a critical review of the manuscript. MAR is a member of the expert panel who helped construct the expert panel recommendations. He also performed a critical review of the manuscript. JAS is a member of the expert panel who helped construct the panel recommendations. He also performed a critical review of the manuscript. VJL helped conceive the study and provided guidance on the format. She is a member of the expert panel who helped construct the panel recommendations. She also performed a critical review of the manuscript.

\section{Acknowledgement}

We thank Lisa A, Marks M.L.S. for her assistance with performing the systematic review. 


\section{Conflict of interest}

\section{None Declared.}

\section{References}

1. Schiller JS, Lucas JW, Peregoy JA. Summary health statistics for U.S. Adults: national health interview survey, 2011. Vital Health Stat 10 2012;(256):1-218.

2. Hastan D, Fokkens WJ, Bachert C, Newson RB, Bislimovska J, Bockelbrink A, et al. Chronic rhinosinusitis in Europe--an underestimated disease. A GA²LEN study. Allergy 2011;66(9):1216-23.

3. Saxby AJ, Pace-Asciak P, Dar Santos RC, Chadha NK, Kozak FK. The rhinological manifestations of women's health. Otolaryngol Head Neck Surg 2013;148(5):717-31.

4. Sobol SE, Wright ED, Frenkiel S. One-year outcome analysis of functional endoscopic sinus surgery for chronic sinusitis. J Otolaryngol 1998;27(5):252-7.

5. Goldstein G, Govindaraj S. Rhinologic issues in pregnancy. Allergy \& Rhinology 2012;3(1):13-5.

6. Howard BE, Lal D. Oral steroid therapy in chronic rhinosinusitis with and without nasal polyposis. Curr Allergy Asthma Rep 2013;13(2):236-43.

7. Orban N, Maughan E, Bleach N. Pregnancyinduced rhinitis. Rhinology 2013;51(2):1119.

8. Rassekh $\mathrm{CH}$, Kinsella JB, Calhoun $\mathrm{KH}$, Maggio WW, Chaljub G, Gourley WK. Skull base allergic fungal sinusitis with abducens palsy in the third trimester. Skull Base Surg 1996;6(4):253-8.

9. Poulopoulos M, Finelli PF. Neurological complications with acute sphenoid sinusitis a surgical emergency? Neurocrit Care 2007;7(2):169-71

10. Rosenfeld RM, Andes D, Bhattacharyya N, Cheung D, Eisenberg S, Ganiats TG, et al. Clinical practice guideline: adult sinusitis. Otolaryngol Head Neck Surg 2007;137(3 Suppl):S1-31.

11. Fokkens WJ, Lund VJ, Mullol J, Bachert C, Alobid I, Baroody F, et al. European Position Paper on Rhinosinusitis and Nasal Polyps 2012. Rhinol Suppl 2012;(23):3pprecedingtableofcontents-1-298.

12. Phillips B, Ball C, Sackett D, Badenoch D, Straus S, Haynes B, et al., editors. Oxford Center for Evidence-based Medicine Levels of Evidence [Internet]. [cited 2015 Mar 31]. Available from: http://www.cebm. net/oxford-centre-evidence-based-medicine-levels-evidence-march-2009/

13. Park-Wyllie L, Mazzotta P, Pastuszak A Moretti ME, Beique L, Hunnisett $L$, et al. Birth defects after maternal exposure to corticosteroids: prospective cohort study and meta-analysis of epidemiological studies. Teratology 2000;62(6):385-92.

14. Schatz M, Zeiger RS, Harden K, Hoffman CC, Chilingar L, Petitti D. The safety of asthma and allergy medications during pregnancy. J Allergy Clin Immunol 1997;100(3):301-6.

15. Perlow JH, Montgomery D, Morgan MA Towers CV, Porto M. Severity of asthma and perinatal outcome. Am J Obstet Gynecol 1992;167(4 Pt 1):963-7.

16. Ellegård EK, Hellgren M, Karlsson NG Fluticasone propionate aqueous nasa spray in pregnancy rhinitis. Clin Otolaryngol Allied Sci 2001;26(5):394-400.

17. Mabry RL. Intranasal steroid injection during pregnancy. South Med J 1980;73(9):1176-9.

18. Murphy VE, Zakar T, Smith R, Giles WB Gibson PG, Clifton VL. Reduced 11 betahydroxysteroid dehydrogenase type 2 activity is associated with decreased birth weight centile in pregnancies complicated by asthma. J Clin Endocrinol Metab 2002;87(4):1660-8.

19. Wendel PJ, Ramin SM, Barnett-Hamm C, Rowe TF, Cunningham FG. Asthma treatment in pregnancy: a randomized controlled study. Am J Obstet Gynecol 1996;175(1):150-4.

20. Dombrowski MP, Schatz M, Wise R, Thom EA, Landon M, Mabie W, et al. Randomized trial of inhaled beclomethasone dipropionate versus theophylline for moderate asthma during pregnancy. Am J Obstet Gynecol 2004;190(3):737-44

21. Norjavaara E, de Verdier MG. Normal pregnancy outcomes in a population-based study including 2,968 pregnant women exposed to budesonide. J Allergy Clin Immunol 2003;111(4):736-42.

22. Briggs G, Freeman R, Yaffe S. Drugs in Pregnancy and Lactation: A Reference Guide to Fetal and Neonatal Risk. 9 ed. Philadelphia: Lippincott Williams \& Wilkins; 2011.

23. Gluck JC, Gluck PA. Asthma controller thera- py during pregnancy. Am J Obstet Gynecol 2005;192(2):369-80.

24. Yau W-P, Mitchell AA, Lin KJ, Werler MM Hernández-Díaz S. Use of decongestants during pregnancy and the risk of birth defects. Am J Epidemiol 2013;178(2):198208.

25. Toll K, Graf P. Phenylpropanolamine's decongestive effect on the nasal mucosa of pregnant women with nasal stuffiness. Rhinology 2006;44(4):274-7.

26. Kariyawasam HK, Rotiroti G, Robinson DS. Sublingual immunotherapy in allergic rhinitis: indications, efficacy and safety. Rhinology 2013;51(1):9-17.

27. Harvey R, Hannan SA, Badia L, Scadding G. Nasal saline irrigations for the symptoms of chronic rhinosinusitis. Cochrane Database Syst Rev 2007;(3):CD006394.

28. Marx GF, Zemaitis MT, Orkin LR. Cerebrospinal fluid pressures during labor and obstetrical anesthesia. Anesthesiology 1961;22:348-54.

29. Schabel JE, Samora GJ, Steinberg ES. Spinal anesthesia for a parturient with an iatrogenic skull base defect and CSF leak. J Clin Anesth 2002;14(8):592-4.

30. Summary of Proposed Rule on Pregnancy and Lactation. www.fda.gov/ Drugs/ DevelopmentApprovalProcess/ DevelopmentResources/Labeling/ ucm093310.htm2009.

\section{Devyani Lal, MD \\ Consultant \\ Department of Otorhinolaryngology- \\ -Head \& Neck Surgery \\ Associate Professor \\ Mayo Clinic College of Medicine \\ 5777 E. Mayo Boulevard \\ Phoenix, AZ 85054 \\ USA}

E-mail: Lal.devyani@mayo.edu 